\title{
Formação em cuidados paliativos oncológicos: desafios para o ensino em saúde na perspectiva de residentes
}

\author{
Training in palliative oncological care: challenges for \\ health teaching from the perspective of residents
}

\author{
Aline Silva Castro', João Paulo Menezes Lima², George Alberto da Silva Dias², \\ Ana Cristina Vidigal Soeiro² \\ 'Universidade do Estado do Pará, Centro de Ciências Biológicas e da Saúde, Programa de Residência \\ Multiprofissional em Saúde, Belém (PA), Brasil. \\ 2Universidade do Estado do Pará, Centro de Ciências Biológicas e da Saúde, Departamento de Ciências do \\ Movimento Humano, Belém (PA), Brasil.
}

\section{RESUMO}

Objetivo: Identificar os desafios enfrentados na formação em cuidados paliativos oncológicos por parte de integrantes do Programa de Residência Multiprofissional em Saúde. Método: Trata-se de um estudo quantitativo, transversal, desenvolvido com residentes do Programa de Residência Multiprofissional em Saúde do Hospital Oncológico Ophir Loyola, em Belém do Pará. A coleta de dados incluiu um questionário semiestruturado, autoaplicado, contendo perguntas relacionadas aos cuidados paliativos e à percepção sobre a estrutura e funcionamento do programa. Os dados foram analisados por meio de estatística descritiva, teste G (aderência) e Qui-quadrado, utilizando-se o software Excel ${ }^{\circledR} 2013$ e Bioestat 5.0. Resultados: Os achados revelam escassez de conteúdos sobre CP durante a graduação. Embora tenham sido apontados aspectos positivos do programa, a articulação entre teoria e prática e o estímulo à realização de estudos e pesquisas científicas, foram identificados como desafios a serem enfrentados diante da intensa demanda de atividades. Conc/usão: Há necessidade de revisão do projeto pedagógico do programa, de modo que os conteúdos teóricos e práticos sobre CP oncológicos sejam distribuídos mais adequadamente ao longo da carga horária do programa. Além disso, o incentivo à produção científica e abertura de espaços de diálogo com a gestão do programa se mostraram necessários ao aprimoramento das atividades de ensino.

PALAVRAS-CHAVE: Cuidados paliativos. Oncologia. Programas de Pós-graduação em Saúde.

Recebido: Fev. 24, 2021 Aceito: Maio 03, 2021

\section{COMO CITAR ESTE ARTIGO}

Castro AS, Lima JPM, Dias GAS, Soeiro ACV. Formação em cuidados paliativos oncológicos: desafios para o ensino em saúde na perspectiva de residentes. Interdisciplinary Journal of Health Education. 2021 Mês-Mês:6(1): 1-9. https://doi.org/10.4322/ijhe.2020.014

\section{CORRESPONDÊNCIA}

Aline Silva Castro

Universidade do Estado do

Pará, Programa de Residência

Multiprofissional em Saúde

Tv. Perebebuí, 2623, Marco, CEP 66087-

662, Belém (PA), Brasil

alinefisio13@hotmail.com

\section{FONTE DE FINANCIAMENTO}

O Projeto foi custeado pelos autores.

\section{ABSTRACT}

Objective: To identify the challenges faced in training in palliative oncology care by members of the Multiprofessional Residency Program in Health. Methods: This is a quantitative, crosssectional study, developed with residents of the Multiprofessional Residency Program in Health at the Hospital Oncológico Ophir Loyola, in Belém do Pará. The data collection included a semistructured, self-administered questionnaire, containing questions related to palliative care and the perception about the structure and functioning of the program. Data were analyzed using descriptive statistics, G test (adherence) and Chi-square, using Excel ${ }^{\circledR} 2013$ and Bioestat 5.0 software. Results: The findings reveal a shortage of content about PC during graduation. Although positive aspects of the program were pointed out, the articulation between theory and practice and the encouragement of scientific studies and research, were identified as challenges to be faced in the face of the intense demand for activities. Conclusion: There is a need to revise the pedagogical project of the program, so that the theoretical and practical contents on oncological PCs are more adequately distributed throughout the program load. In addition, encouraging scientific production and opening spaces for dialogue with the program management proved necessary to improve teaching activities.

KEYWORDS: Palliative care. Oncology. Postgraduate Health Programs.

\section{CONFLITO DE INTERESSE}

Os autores declararam não

haver conflitos de interesse. 


\section{Introdução}

Atualmente, o câncer é a segunda principal causa de morte no mundo, com uma estimativa crescente de novos casos na população mundial. No cenário brasileiro, foram realizados diversos investimentos voltados à ampliação da rede de atenção ao câncer, com oferta de exames diagnósticos e tratamento. Porém, este aprimoramento ainda é insuficiente para atender às demandas epidemiológicas nacionais, as quais incluem, dentre outros, a formação e aprimoramento de profissionais com expertise na área ${ }^{1,2}$.

Concomitantemente ao progressivo fortalecimento da assistência oncológica no país, os Cuidados Paliativos (CP) oncológicos também têm se expandindo, não só como um campo especial da atenção oncológica, mas em seu sentido mais amplo, como uma filosofia de cuidado voltada à minimização da dor e do sofrimento humano. Trata-se de uma importante abordagem terapêutica para o enfrentamento de doenças potencialmente ameaçadoras da vida, e um conteúdo indispensável a ser contemplado na formação em saúde ${ }^{3,4}$.

Considerando tais peculiaridades, é imprescindível que todos os profissionais da saúde tenham acesso a conhecimentos sobre o tema durante seu processo de formação. Em se tratando daqueles que escolhem os CP como área de atuação, o conhecimento das ferramentas teóricas e metodológicas, incluindo educação especializada na área, é condição insubstituível para uma atuação qualificada e eficaz junto aos pacientes ${ }^{5}$.

No Brasil, os CP surgiram como campo de especialização apenas recentemente, haja vista que os profissionais pioneiros nessa abordagem, tiveram uma formação autodidata. Somente a partir do ano de 2010, começaram a surgir horizontes para o estudo da temática, por meio de novos modelos de especialidade em diversas áreas da saúde, a exemplo dos programas de residência multiprofissional ${ }^{6}$.

Nesse contexto, a residência multiprofissional representa uma opção de pósgraduação baseada na articulação ensino e serviço, que busca formar profissionais capacitados a lidar com os desafios no campo da saúde, com base nas necessidades e particularidades loco-regionais. Trata-se de uma modalidade de ensino em saúde que visa à concretização e sustentação das políticas públicas do Sistema Único de Saúde (SUS), por meio de uma abordagem problematizadora e transformadora da realidade. Os programas de residência representam uma oportunidade para sensibilizar e qualificar equipes, sobretudo em relação a temas pouco explorados nos currículos acadêmicos de graduação, a exemplo da temática dos $\mathrm{CP}^{7,8}$.

Diversos aspectos estão envolvidos na busca por formação em $\mathrm{CP}$, incluindo a sensibilidade e motivação, as habilidades técnico-assistenciais, as quais somadas às características pessoais, constituem atributos importantes para o cuidado paliativo,10. O profissional que opta por explorar este âmbito do cuidado enfrenta diversos desafios, os quais precisam ser compreendidos, incluindo nesse cenário os impactos dessa experiência em sua vida pessoal e profissional9,11,12.

Considerando a relevância do tema no âmbito das políticas e práticas em saúde, o presente estudo foi projetado com o objetivo de identificar os desafios enfrentados na formação em CP oncológicos por parte de integrantes do Programa de Residência Multiprofissional em Saúde.

\section{Metodologia}

A pesquisa teve início após aprovação de Comitê de Ética e Pesquisa, sob parecer $\mathrm{n}^{\circ}$ 4.144.634, e foi realizada no Hospital Ophir Loyola (HOL), reconhecido como um Centro de Assistência de Alta Complexidade em Oncologia (CACON), referência no tratamento oncológico no Pará. Participaram do estudo os residentes vinculados ao Programa de Residência Multiprofissional em Saúde da Universidade do Estado do Pará (UEPA), com área de concentração em Oncologia/Cuidados Paliativos, 
selecionados por meio da amostragem não probabilística, por conveniência. Todos os participantes concordaram com a inclusão no estudo mediante a assinatura do Termo de Consentimento Livre e Esclarecido (TCLE).

A coleta de dados ocorreu em agosto de 2020, por meio da aplicação de um questionário semiestruturado, contendo 31 questões elaboradas pelos pesquisadores. A primeira parte do instrumento continha informações sobre o contato prévio com os $\mathrm{CP}$, e também sobre os fatores que influenciaram a escolha pela temática, incluindo a motivação para atuação na área. A segunda parte do questionário foi organizada de modo a identificar os desafios encontrados em relação à dinâmica de funcionamento do programa, incluindo a organização das atividades e o relacionamento com a coordenação, tutores e preceptores.

A fim de garantir a privacidade, os participantes foram direcionados à biblioteca da instituição onde recebiam o questionário autoaplicável. A pesquisadora permanecia no local e ficava disponível para esclarecimentos e recolhimento do material após preenchimento completo. Os questionários eram então codificados e armazenados para posterior análise. Para entrada dos dados e análise estatística, adotou-se o software Excel® 2013 e BioEstat 5.0. As variáveis numéricas foram apresentadas por meio de medidas de tendência central (média) e dispersão (desvio padrão), e os testes G (Aderência) e Qui-quadrado para avaliar a significância dos dados. Adotouse o nível alfa de significância de $5 \%(p<0,05)$.

\section{Resultados}

De um total de 25 residentes, participaram da pesquisa 24 integrantes da residência, dos quais a maioria era do sexo feminino $(n=20 ; 83,33 \%)$, com média de idade de $26,33 \pm 2,86$ anos e 1,96 $\pm 1,26$ anos de formados. Com relação à profissão, participaram 3 (12,50\%) Fonoaudiólogos, 3 (12,50\%) Fisioterapeutas, 4 (16,66\%) Enfermeiros, 4 (16,66\%) Terapeutas Ocupacionais, 3 (12,50\%) Nutricionistas, 3 (12,50\%) Psicólogos e $3(12,50 \%)$ Assistentes Sociais. Destes, 13 (54,16\%) estavam no primeiro ano de residência e $11(45,83 \%)$ no segundo ano de residência. No que diz respeito às atividades acadêmicas e/ou profissionais realizadas antes do ingresso na residência, a maioria ( $\mathrm{n}=11 ; 45,33 \%)$ não possuía experiência prévia (Tabela 1$)$.

Quando indagados sobre o contato com a temática durante a graduação, foi observado, de forma significante, que os residentes tiveram contato com o tema somente algumas vezes $(\mathrm{p}=0,0003)$. Nas respostas, foi possível constatar, de forma significante $(\mathrm{p}=0,002)$, a escassez desta temática como disciplina específica, estágio obrigatório e extracurricular. Conforme demonstrado na Tabela 2, a abordagem do tema ocorreu com maior frequência durante aulas expositivas e eventos científicos, mesmo não apresentando resultados significantes $(\mathrm{p}=0,813)$.

Com relação à motivação para a escolha do programa na área de $\mathrm{CP}$, a maioria referiu a remuneração, enriquecimento do currículo profissional, experiência prática, título de especialista e aprendizado, como fatores motivacionais, mesmo sem significância estatística. No entanto, o número de vagas não foi um fator motivador, com resultado significante $(\mathrm{p}=0,0005)$. No que se refere à motivação para permanência no programa, variáveis como o interesse pelo tema, remuneração, experiência prática, realização pessoal, título de especialista e aprendizado foram os mais prevalentes. Além disso, a equipe de trabalho não despontou como um fator motivador com resultados significantes $(\mathrm{p}<0,0001)$.

No que concerne à dedicação a atividades científicas $(\mathrm{p}=0,0003)$, estímulo à participação em eventos científicos $(\mathrm{p}=0,001)$, incentivo à produção acadêmica $(\mathrm{p}=0,001)$, e articulação entre teoria e prática $(\mathrm{p}=0,0035)$, a maioria dos participantes respondeu "algumas vezes", com resultados significantes. 
Tabela 1. Caracterização dos residentes que participaram do estudo.

\begin{tabular}{|c|c|c|}
\hline \multirow{2}{*}{ Variáveis } & \multicolumn{2}{|c|}{ Participantes $(n=24)$} \\
\hline & Média & DP \\
\hline Idade (anos) & 26,33 & $\pm 2,86$ \\
\hline \multirow[t]{2}{*}{ Tempo de graduação (anos) } & 1,96 & $\pm 1,26$ \\
\hline & n & $\%$ \\
\hline \multicolumn{3}{|l|}{ Sexo } \\
\hline Feminino & 20 & 83,33 \\
\hline Masculino & 4 & 16,66 \\
\hline \multicolumn{3}{|l|}{ Profissão } \\
\hline Fonoaugiólogo & 3 & 12,50 \\
\hline Fisioterapeuta & 3 & 12,50 \\
\hline Enfermeiro & 4 & 16,66 \\
\hline Terapeuta Ocupacional & 4 & 16,66 \\
\hline Nutricionista & 3 & 12,50 \\
\hline Psicólogo & 3 & 12,50 \\
\hline Assistente Social & 3 & 12,50 \\
\hline \multicolumn{3}{|l|}{ Ano da residência } \\
\hline $1^{\circ}$ ano & 13 & 54,16 \\
\hline $2^{\circ}$ ano & 11 & 45,83 \\
\hline \multicolumn{3}{|c|}{ Atividades acadêmicas ou profissionais prévias } \\
\hline Não possui & 11 & 45,33 \\
\hline Emprego formal & 7 & 29,16 \\
\hline Residência & 3 & 12,50 \\
\hline Especialização & 2 & 8,33 \\
\hline Mestrado & 1 & 4,16 \\
\hline
\end{tabular}

$\mathrm{n}$ = frequência absoluta; \% = frequência relativa; DP = desvio padrão. Fonte: Dados da pesquisa, 2021.

Tabela 2. Distribuição da frequência de repostas sobre o contato com a temática a graduação.

\begin{tabular}{lccc}
\multicolumn{1}{c}{ Variáveis } & $\begin{array}{c}\text { Sim } \\
\mathbf{n}(\mathbf{\%})\end{array}$ & $\begin{array}{c}\text { Não } \\
\mathbf{n}(\mathbf{\%})\end{array}$ & p-valor \\
\hline Contato com a temática CP na graduação & & & \\
\hline Disciplina específica & $2(11)$ & $16(89)$ & $\mathbf{0 , 0 0 2}$ \\
\hline Aula expositiva & $10(56)$ & $08(44)$ & 0,813 \\
\hline Estágio Obrigatório & $2(11)$ & $16(89)$ & $\mathbf{0 , 0 0 2}$ \\
\hline Estágio Extracurricular & $2(11)$ & $16(89)$ & $\mathbf{0 , 0 0 2}$ \\
\hline Eventos Científicos & $8(44)$ & $10(56)$ & 0,813 \\
\hline Outros & $2(11)$ & $16(89)$ & $\mathbf{0 , 0 0 2}$ \\
\hline
\end{tabular}

Teste do Qui-quadrado, $\mathrm{p}<0,05$. Fonte: Dados da pesquisa, 2021.

Em relação ao processo avaliativo, $88 \%(n=21)$ responderam que "nunca" avaliaram $\mathrm{o}$ tutor, com resultado significante $(\mathrm{p}=0,0005)$. Embora sem significância estatística, a maioria também respondeu que nunca avaliou o preceptor $(\mathrm{p}=0.0662)$. Por fim, quando investigada a frequência com que os residentes eram avaliados, verificou-se a regularidade mensal para $83 \%$ dos participantes $(p<0,0001)$. 
Quando perguntados sobre o interesse em seguir atuando na área, 75\% responderam que têm interesse em permanecer atuando na atenção oncológica (Tabela 3). Em relação à atuação em $\mathrm{CP}$, a taxa de respostas positivas foi de $79 \%$, respectivamente $(\mathrm{p}<0,0001)$.

Quando foram questionados acerca das repercussões da formação em CP no cotidiano de vida, a maioria dos participantes respondeu que as atividades repercutiram "bastante" na vida pessoal $(63 \%, \mathrm{p}=0,0003)$, como também, no campo profissional $(58 \%, \mathrm{p}=0,0003)$ (Tabela 4$)$.

No que tange ao ambiente relacional, os participantes foram solicitados a avaliar o relacionamento com os diferentes atores do programa. Do total, $42 \%$ participantes consideraram a relação com a coordenação como "regular" ( $\mathrm{p}=0,022)$, e 33\% avaliaram a relação com os tutores também como "regular" ( $p=0,002)$. Entretanto, 58\% afirmaram ter uma "boa" relação com os preceptores $(\mathrm{p}=0,0004)$, refletindo também uma avaliação positiva do papel do preceptor no programa $(42 \%, p=0,01)$. Quanto ao instrumento utilizado para avaliar o desempenho dos residentes, $42 \%$ consideraram ser "bom" ( $\mathrm{p}=0,0368)$.

Por fim, foi avaliada a satisfação dos residentes com o Programa de Residência. Na opinião de $75 \%$ dos participantes, o programa de residência atendeu parcialmente às expectativas depositadas na formação $(p<0,0001)$. Foi observado que a maioria $(50 \%$, $\mathrm{p}=0,01$ ) respondeu "ruim" com relação à abordagem dos conteúdos teóricos, bem como para o tempo disponível para estudo dos conteúdos (54\%, $\mathrm{p}=0,03)$. Ademais, $38 \%(\mathrm{p}=0,03)$ respondeu "regular" para a quantidade de carga horária prática.

\section{Discussão}

Atualmente, a inclusão dos CP nas grades curriculares nacionais é marcada por desafios que atravessam as várias categorias profissionais. Como resultado das lacunas existentes, ocorrem prejuízos na aquisição de conhecimentos, habilidades e competências para a atuação em $\mathrm{CP}$, realidade que interfere diretamente nas ações de cuidado em saúde, como aponta Bastos et al. ${ }^{13}$.

Tabela 3. Distribuição da frequência de repostas sobre o desejo de atuar em Oncologia e CP.

\begin{tabular}{lcccc} 
& \multicolumn{3}{c}{ Respostas } & p-valor \\
\cline { 2 - 4 } \multicolumn{1}{c}{ Perguntas } & $\begin{array}{c}\text { Sim } \\
\text { n (\%) }\end{array}$ & $\begin{array}{c}\text { Não } \\
\text { n (\%) }\end{array}$ & $\begin{array}{c}\text { Não sei } \\
\text { responder } \\
\text { n (\%) }\end{array}$ & \\
Pretende atuar em Oncologia? & $18(75)$ & $2(8)$ & $4(17)$ & $\mathbf{0 , 0 0 0 1}$ \\
\hline Pretende atuar em CP? & $19(79)$ & $1(4)$ & $4(17)$ & $<\mathbf{0 , 0 0 0 1}$ \\
\hline
\end{tabular}

Teste G (Aderência), p<0,05. Fonte: Dados da pesquisa, 2021.

Tabela 4. Distribuição da frequência de repostas quanto às repercussões na vida profissional e pessoal.

\begin{tabular}{|c|c|c|c|c|c|c|}
\hline \multirow{2}{*}{ Perguntas } & Nenhuma & Pouca & $\begin{array}{c}\text { Mais ou } \\
\text { menos }\end{array}$ & Bastante & Extrema & \multirow{2}{*}{ p-valor } \\
\hline & n (\%) & n (\%) & n (\%) & n (\%) & n (\%) & \\
\hline $\begin{array}{l}\text { Repercussão dos CP na } \\
\text { vida pessoal }\end{array}$ & - & - & $4(17)$ & $15(63)$ & $5(21)$ & 0,0003 \\
\hline $\begin{array}{l}\text { Repercussão dos CP na } \\
\text { vida profissional futura }\end{array}$ & - & $1(4)$ & $1(4)$ & $14(58)$ & $7(29)$ & 0,0003 \\
\hline
\end{tabular}

(-) Dados numéricos igual a zero. Testes G (Aderência), p<0,05. Fonte: Dados da pesquisa, 2021. 
Apesar do foco da pesquisa estar direcionado ao Programa de Residência Multiprofissional em CP oncológicos, o contexto da formação na área revela fragilidades metodológicas e pedagógicas que antecedem o ingresso dos residentes no programa, sendo esse fato evidenciado no escasso contato prévio dos participantes com a temática.

Cabe ressaltar que os resultados apontam a necessidade de revisão e atualização das diretrizes curriculares nacionais de cada área específica, tendo como objetivo a inclusão e aprofundamento de conteúdos sobre $\mathrm{CP}$ nas grades curriculares. Além disso, os resultados também revelam a importância de criar estratégias voltadas ao incentivo da produção científica na área, em especial quando se consideram os avanços da temática em âmbito nacional ${ }^{14}$. É preciso que as instituições, ao receberem os residentes, criem espaços de fomento à pesquisa e produção de artigos/trabalhos na área de $\mathrm{CP}$, uma iniciativa que também fortalece as ações educacionais realizadas no âmbito institucional.

Cabe ressaltar que o número reduzido de vagas ofertadas, foi identificado como um fator desmotivador para o ingresso no programa. Tal resultado pode ser analisado sob a ótica da heterogeneidade geográfica, no que diz respeito à distribuição e implantação dos programas de Residência Multiprofissional, pelo Ministério da Saúde (MS). O Programa segue as diretrizes da Portaria Interministerial $n^{\circ} 1077 / 2009$, a qual dispõe acerca da criação de bolsas para Residência Multiprofissional em Saúde $(\mathrm{RMS})^{15}$, entretanto, as ações previstas não têm ocorrido de forma homogênea no país.

A despeito dos avanços, foram pontuadas as desigualdades territoriais, sendo as regiões Norte e Centro-Oeste as que apresentam menor concentração de programas de RM, apesar dos movimentos de expansão ocorridos desde o ano de $2015^{16}$. Tal resultado representa um desafio no fortalecimento do princípio da equidade no SUS, e consequentemente, na oferta de serviços de saúde qualificados que atendam às demandas populacionais. Assim, a limitação das vagas, também acarreta prejuízos à efetiva ampliação das ações, haja vista que reduz a quantidade de profissionais qualificados para atuar em CP.

A interação com a equipe de trabalho foi apontada pelos participantes como um fator que não os motiva a permanecer no programa de residência, mesmo tratandose de uma formação com caráter multiprofissional e centrada na articulação ensino e serviço. Esta dificuldade, expressa inclusive no distanciamento dos residentes com a gestão do Programa, incluindo a coordenação e tutores, pode estar associada em certa medida, às dificuldades para realizar um trabalho articulado e coletivo, que privilegie a integração nas ações de atenção e gestão.

Além disso, também pode ser uma árdua tarefa a mudança de práticas já solidificadas na instituição, daí a importância de ampliar os espaços de escuta e diálogo entre os residentes e as equipes de trabalho, no alcance de transformações importantes e desejáveis nos processos de formação em saúde, rumo a práticas mais democráticas ${ }^{17}$. O convívio com as equipes de saúde é parte importante do processo de formação dos residentes e pressupõe a existência de um ambiente acolhedor, onde os mesmos possam ser vistos como integrantes da equipe, o que significa valorizar sua participação no desenvolvimento das atividades.

Ao avaliarem o relacionamento com os atores do programa, os participantes apontaram a falta de preparo da equipe de saúde em relação ao acolhimento dos residentes. Este achado reforça a necessidade de capacitação não só dos preceptores, como dos demais envolvidos neste processo de formação e que atuam nos locais de prática. A integração ensino e serviço promove novas interações no trabalho em equipe, acarretando troca de experiências e aprendizado, construídas na diversidade e colaboração dos envolvidos, estimulando práticas mais enriquecedoras de aprendizagem significativa ${ }^{18}$.

Tal modelo de aprendizagem propõe que o residente exerça um papel ativo e protagonista, articulando o conhecimento adquirido às experiências vivenciadas no serviço. Por isso, a RMS deve dispor de metodologias ativas que visem uma formação 
que integre os saberes de todos os núcleos profissionais, aproximando os diferentes sujeitos envolvidos no SUS ${ }^{19}$. Nesse sentido, é possível afirmar que a integralidade das práticas em saúde depende também da capacidade de articulação das equipes por meio de decisões e ações compartilhadas, nas quais o residente também deve ser incluído.

Um dos objetivos dos programas de RMS é possibilitar uma qualificação profissional diferenciada e baseada em princípios da Política Nacional de Educação Permanente em Saúde (PNEPS) e inovação no $\mathrm{SUS}^{20}$, entretanto, o pouco estímulo à participação em atividades científicas, a falta de incentivo à produção acadêmica e a frágil articulação entre teoria e prática, constituem desafios ao ensino em saúde.

O papel da educação permanente nos programas de RMS em CP oncológicos deve oportunizar o desenvolvimento de habilidades teórico-práticas que possibilitam a atuação dentro de cenários complexos, com destaque para as situações de comunicação de más notícias, terminalidade da vida, manejo da dor, preservação da funcionalidade, dentre outras ${ }^{13,21}$. Entretanto, também é necessário considerar o campo da gestão em saúde, em função da necessidade de buscar o aprimoramento e consolidação das redes assistenciais e linhas de cuidado em $\mathrm{CP}^{22}$.

Ao refletir sobre a importância da educação permanente em saúde, torna-se fundamental considerar também o papel dos diferentes atores implicados no contexto em questão, tais como tutores, preceptores e coordenação do programa. Nessa perspectiva, considerar o papel do corpo docente-administrativo requer uma análise da dimensão técnico-operacional, a qual compreende a organização dos processos de trabalho, estruturação pedagógico-assistencial e dinâmica relacional, com o intuito de identificar potencialidades e eventuais fragilidades a serem discutidas de forma coletiva ${ }^{23}$.

A fragilidade em relação aos mecanismos de avaliação de tutores e preceptores merece ser destacada, pois constitui uma estratégia importante na formulação de diagnósticos, na análise do desempenho, na instauração de processos de mudanças, assim como no dialogismo e na avaliação das experiências proporcionadas pelo programa ${ }^{24}$. Ademais, serve como um mecanismo de acompanhamento e monitoramento das atividades, oportunizando assim a possibilidade de aprimorar o processo ensino-aprendizagem.

A dificuldade nos processos avaliativos dos atores envolvidos nos programas de RMS também foi explorada no estudo de Vasconcelos et al. ${ }^{25}$, o qual identificou problemas nos modelos propostos, valorizando a importância da autoavaliação no aperfeiçoamento institucional. Assim, instrumentos capazes de examinar a formação em CP oncológicos devem avaliar os papéis e atitudes esperadas dos atores, assim como as competências e habilidades requeridas nos campos de atuação.

Cabe ressaltar que a relação dos residentes com a coordenação e tutores foi classificada como "regular", o que indica a necessidade de investigar os principais impasses e desencontros experimentados, incluindo uma clara definição de papéis, com o objetivo de adequar soluções e medidas viáveis para resolução dos principais nós críticos.

O distanciamento da coordenação-docente do programa com os residentes também foi citada no artigo de Miolo e Fedosse ${ }^{26}$. Na opinião dos autores, tais dificuldades ocasionam entraves na articulação do eixo ensino-serviço, uma vez que as demandas dos cenários práticos e dos processos de ensino-aprendizagem não são discutidas, e consequentemente, examinadas, dificultando mudanças e melhorias no ensino em serviço. Ademais, é importante destacar que um dos atores com o papel-chave no diálogo entre residentes e os cenários de prática é o(a) tutor(a). Entretanto, achados na literatura apontam dificuldades nessa função, como a insuficiência de carga horária para o exercício da tutoria e os tensionamentos envolvidos na parceria com os atores dos cenários de prática ${ }^{27}$.

Os programas de residência comportam diferentes aspectos desafiadores, e a excessiva carga horária de trabalho é frequentemente apontada como uma das 
principais dificuldades. Tal realidade contribui para que alguns residentes se sintam prejudicados na aquisição de conteúdos teóricos, e na realização de trabalhos e pesquisas científicas, como resultado da grande demanda de atividades na rotina do serviço. No entanto, mesmo que a prática em serviço seja priorizada nesses ambientes de aprendizagem, é necessária a integração entre as ações de ensino, pesquisa e assistência, o que influenciará significativamente no aprendizado dos residentes ${ }^{23}$.

Cabe destacar que a excessiva carga horária de trabalho pode ser considerada um fator de estresse para os residentes, ocasionando desgaste físico e mental, como afirmam Silva e Moreira ${ }^{28}$. Os autores sinalizam o burnout de residentes como uma pauta que merece ênfase, em virtude de constituir um entrave ao processo formativo e à aprendizagem significativa. Em se tratando dos $\mathrm{CP}$, o constante convívio com a dor, o sofrimento e a morte, pode repercutir na saúde mental dos residentes, com impactos na vida pessoal e no desempenho das atividades profissionais.

Tais achados sugerem a necessidade de uma constante avaliação e revisão dos métodos de aprendizagem utilizados nesses locais. Do mesmo modo, a disponibilização de espaços de escuta e reflexão das experiências vividas, é uma iniciativa importante também para o aprimoramento das habilidades socioemocionais dos residentes. Logo, metodologias e estratégias de aprendizagem baseadas em problemas, impulsionadas pelo cotidiano e pela prática assistencial, são importantes para estimular a formação crítica dos residentes diante de uma realidade complexa e dinâmica ${ }^{29}$.

\section{Conclusão}

Os avanços na área dos CP oncológicos caminham em paralelo ao aprimoramento das propostas de ensino em saúde, com destaque para os programas de residência multiprofissional. Nesse sentido, os achados do presente estudo possibilitaram identificar alguns dos principais desafios vivenciados por residentes da área de CP oncológicos e apontam a necessidade de revisão do projeto pedagógico do programa, tendo em vista a necessidade de alinhamento das práticas de ensino-aprendizagem com as premissas da educação permanente.

De modo geral, constatou-se a importância de adequação da carga horária nos processos de trabalho, de modo que os residentes possam aprimorar a articulação entre os conhecimentos teóricos e práticos sobre os CP oncológicos. Do mesmo modo, os resultados apontaram a necessidade de maior incentivo à produção científica, aprimoramento do ambiente relacional e abertura de espaços de diálogo com a gestão do programa.

O quantitativo de participantes e a metodologia utilizada constituem algumas limitações do estudo, haja vista que a inclusão de dados qualitativos provenientes de narrativas dos participantes ampliaria o alcance dos achados. Logo, embora o estudo tenha contribuído para dar maior visibilidade ao tema, vale ressaltar a importância de novas pesquisas relacionadas aos $\mathrm{CP}$ oncológicos, e que considerem as particularidades da Região Norte, objetivando a melhoria nos processos de ensino em saúde, particularmente no que concerne à formação em cuidados paliativos.

\section{Referências}

1. Migowski A, Atty ATM, Tomazelli JG, Dias MBK, Jardim BC. A atenção oncológica e os 30 anos do Sistema Único de Saúde. Rev Bras Canc. 2018;64(2):247-50.

2. Brasil. Instituto Nacional do Câncer. Onde tratar pelo SUS. Brasília: Ministério da Saúde; 2019.

3. Poletto S, Bettinelli LA, Santin JR. Vivências da morte de pacientes idosos na prática médica e dignidade humana. Rev Bioet. 2016;24(3):590-5. http://dx.doi.org/10.1590/1983-80422016243158.

4. McClung JA. End-of-life care in the treatment of heart failure in older adults. Heart Fail Clin. 2017;13(3):633-44. http:// dx.doi.org/10.1016/j.hfc.2017.02.014. PMid:28602377.

5. Pacheco CL, Goldim JR. Percepções da equipe interdisciplinar sobre cuidados paliativos em oncologia pediátrica. Rev Bioet. 2019;27(1):67-75. http://dx.doi.org/10.1590/1983-80422019271288.

6. Krasilcic S. Ensino de cuidados paliativos na graduação: objetivos, situação atual e desafios. Rev Cuid Pal. 2015;1(3):18-21.

7. Silva LB. Residência Multiprofissional em Saúde no Brasil: alguns aspectos da trajetória histórica. R Katál. 2018;21(1):2009. http://dx.doi.org/10.1590/1982-02592018v21n1p200. 

GEP News. 2019;2(2):252-60.

9. Fonseca A, Geovanini F. Cuidados paliativos na formação do profissional da área de saúde. Rev Bras Educ Med. 2013;37(1):1205. http://dx.doi.org/10.1590/S0100-55022013000100017.

10. Costa AP, Poles K, Silva AE. Formação em cuidados paliativos: experiência de alunos de medicina e enfermagem. Interface Comun Saude Educ. 2016;20(59):1041-52. http://dx.doi.org/10.1590/1807-57622015.0774.

11. Almeida CSL, Marcon SS, Matsuda LM, Kantorski LP, Paiva BSR, Sales CA. Atuação de um serviço de cuidados paliativo hospitalar: avaliação de quarta geração. Rev Bras Enferm. 2019;72(2):401-8. PMid:31017200.

12. Cezar VS, Waterkemper R, Rabin EG, Castilho RK, Reys KZ. Educação permanente em cuidados paliativos: uma proposta de pesquisa-ação. Rev Online Pesq. 2019;11(2):324-32. http://dx.doi.org/10.9789/2175-5361.2019.v11i2.324-332.

13. Bastos BR, Fonseca ACG, Pereira AKS, Souza e Silva LC. Formação dos profissionais de saúde na comunicação de más notícias em cuidados paliativos oncológicos. Rev Bras Cancerol. 2016;62(3):263-6. http://dx.doi.org/10.32635/2176-9745. RBC.2016v62n3.342.

14. Pineli PP, Krasilcic S, Suzuki FA, Maciel MGS. Cuidado paliativo e diretrizes curriculares: inclusão necessária. Rev Bras Educ Med. 2016;40(1):540-6. http://dx.doi.org/10.1590/1981-52712015v40n4e01182015.

15. Brasil. Ministério da Saúde. Portaria interministerial n 1.077, de 12 de novembro de 2009. Dispõe sobre a Residência Multiprofissional em Saúde e a Residência em Área Profissional da Saúde, e institui o Programa Nacional de Bolsas para Residências Multiprofissionais e em Área Profissional da Saúde e a Comissão Nacional de Residência Multiprofissional em Saúde. Diário Oficial da União, Brasília, DF, 2009.

16. Sarmento LF, França T, Medeiros KR, Santos MR, Ney MS. Distribuição regional da oferta de formação na modalidade Residência multiprofissional em saúde. Saúde Debate. 2017;41(113):415-24. http://dx.doi.org/10.1590/0103-1104201711306.

17. Silva CA, Dalbello-Araujo M. Programa de Residência Multiprofissional em Saúde: o que mostram as publicações. Saúde Debate. 2019;43(123):1240-58. http://dx.doi.org/10.1590/0103-1104201912320.

18. Arnemann CT, Kruse MHL, Gastaldo D, et al. Práticas exitosas dos preceptores de uma residência multiprofissional: interface com a interprofissionalidade. Interface Comun Saude Educ. 2018;22(2, Supl. 2):1635-46. http://dx.doi. org/10.1590/1807-57622017.0841.

19. Casanova IA, Batista NA, Ruiz-Moreno L. Formação para o trabalho em equipe na residência multiprofisssional em saúde. ABCS Health Sci. 2015;40(3):229-33. http://dx.doi.org/10.7322/abcshs.v40i3.800.

20. Brasil. Ministério da Saúde. Política de educação edesenvolvimento para o SUS: caminhos para a educação permanente e pólos de educação permanente em saúde. Brasília: Ministério da Saúde; 2004.

21. Silva CT, Terra MG, Kruse MHL, Camponogara S, Xavier MS. Residência multiprofissional como espaço intercessor para a educação permanente em saúde. Texto Contexto Enferm. 2016;25(1):e2760014. http://dx.doi.org/10.1590/0104-0707201600002760014.

22. Carvalho GAFL, Menezes RMP, Enders BC, Teixeira GA, Dantas DNA, Oliveira DRC. Significados atribuídos por profissionais de saúde aos cuidados paliativos no contexto da Atenção Primária. Texto Contexto Enferm. 2018;27(2):e5740016. http:// dx.doi.org/10.1590/0104-070720180005740016.

23. Silva LS, Natal S. Residência Multiprofissional em Saúde: análise da implantação de dois programas pela Universidade Federal de Santa Catarina Brasil. Trab Educ Saúde. 2019;17(3):e0022050. http://dx.doi.org/10.1590/1981-7746-sol00220.

24. Melo MC, Queluci GC, Gouvêa MV. Problematizando a residência multiprofissional em oncologia: protocolo de ensino prático na perspectiva de residentes de enfermagem. Rev Esc Enferm USP. 2014;48(4):706-14. http://dx.doi.org/10.1590/ S0080-623420140000400019. PMid:25338253.

25. Vasconcelos MIO, Souza FL, Lira GV, Dias MSA, Silva GSN. Avaliação de programas de residência multiprofissional em saúde da família por indicadores. Trab Educ Saúde. 2015;13(2, Supl. 2):53-77. http://dx.doi.org/10.1590/1981-7746-sip00080.

26. Miolo SB, Fedosse E. Tutoria e preceptoria em programas de residência multiprofissional na atenção básica: um diálogo necessário. Res Soc Dev. 2020;9(3):88932477.

27. Andrade SRD, Boehs AE, Boehs CGE. Percepções de enfermeiros docentes e assistenciais sobre a parceria ensino-serviço em unidades básicas de saúde. Interface Comun Saude Educ. 2015;19(1):537-47. http://dx.doi.org/10.1590/1807-57622014.0277.

28. Silva MRB, Moreira SNT. Estresse e residência multiprofissional em saúde: compreendendo significados no processo de formação. Rev Bras Educ Med. 2019;43(4):157-66. http://dx.doi.org/10.1590/1981-52712015v43n4rb20190031.

29. Torres RBS, Barreto ICHC, Carvalho JB. Conflitos e avanços na implementação de uma Residência Integrada em Saúde com ênfase em Cancerologia. ABCS Health Sci. 2015;40(3):370-6. http://dx.doi.org/10.7322/abcshs.v40i3.824.

\section{Contribuição dos autores}

Aline Silva Castro participou da construção do desenho do trabalho, interpretação dos dados da pesquisa, da redação e revisão crítica com contribuição intelectual e na revisão final do manuscrito. João Paulo Menezes de Lima participou da interpretação dos dados da pesquisa, na redação e revisão crítica com contribuição intelectual e na revisão final do manuscrito. George Alberto da Silva Dias participou da interpretação dos dados da pesquisa, da revisão crítica com contribuição intelectual e na revisão final do manuscrito. Ana Cristina Vidigal Soeiro participou da construção do desenho do trabalho, interpretação dos dados da pesquisa, na redação e revisão crítica com contribuição intelectual e na revisão final do manuscrito. 